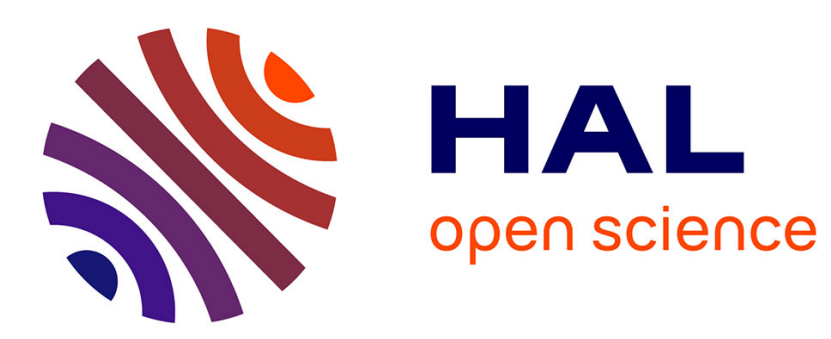

\title{
A Mathematical Model of Mast Cell Response to Acupuncture Needling
}

Yannick Deleuze

\section{To cite this version:}

Yannick Deleuze. A Mathematical Model of Mast Cell Response to Acupuncture Needling. Comptes rendus de l'Académie des sciences. Série I, Mathématique, 2013, 351 (3-4), pp.101-105. 10.1016/j.crma.2013.02.003 . hal-00795833

\section{HAL Id: hal-00795833 https://hal.sorbonne-universite.fr/hal-00795833}

Submitted on 1 Mar 2013

HAL is a multi-disciplinary open access archive for the deposit and dissemination of scientific research documents, whether they are published or not. The documents may come from teaching and research institutions in France or abroad, or from public or private research centers.
L'archive ouverte pluridisciplinaire HAL, est destinée au dépôt et à la diffusion de documents scientifiques de niveau recherche, publiés ou non, émanant des établissements d'enseignement et de recherche français ou étrangers, des laboratoires publics ou privés. 


\title{
A mathematical model of mast cell response to acupuncture needling *
}

\author{
Yannick Deleuze ${ }^{\mathrm{a}, \mathrm{b}}$ \\ ${ }^{a}$ UPMC Univ Paris 06, UMR 7598, Laboratoire Jacques-Louis Lions, 4 place Jussieu, F-75005, Paris, France \\ ${ }^{\mathrm{b}}$ Department of Engineering Science and Ocean Engineering, National Taiwan University, No. 1, Sec. 4, Roosevelt Rd., \\ Da-an District, Taipei 10617, Taiwan, R.O.C.
}

\begin{abstract}
We introduce a new model of mast cell response to acupuncture needling based on the Keller-Segel model for chemotaxis. The needle manipulation induces the release of a chemoattractant by the mast cells. We show, in a simplified case, that blow-up of the solution occurs in finite time for large initial data concentrated around the acupoint. In those conditions, blow-up is the result of aggregation of cells and could indicate the efficiency of the acupuncture manipulation of the needle at one acupoint. To cite this article: Y. Deleuze, C. R. Acad. Sci. Paris, Ser. I 340 (2005).
\end{abstract}

\section{Résumé}

Un modèle mathématique de la réponse des mastocytes à la manipulation d'une aiguille d'acupuncture. Nous présentons un nouveau modèle de la réponse des mastocytes à la manipulation d'une aiguille d'acupuncture basé sur le modèle de chimiotaxie de type Keller-Segel. La manipulation de l'aiguille induit la libération du chimioattractant par les mastocytes. Nous montrons, dans un système simplifié, que la solution devient singulière en un temps fini pour des conditions initiales suffisamment grandes et concentrées autour du point acupuncture. Dans ces conditions, l'explosion de la solutions résulte de l'agrégation des cellules et pourrait mesurer l'efficacité de la manipulation de l'aiguille sur le point d'acupuncture. Pour citer cet article: Y. Deleuze, C. R. Acad. Sci. Paris, Ser. I 340 (2005).

\section{Introduction}

During acupuncture treatment hair-thin needles are inserted in the skin via manual lifting and thrusting or rotating at specific points on the body, called acupoints [1]. The needle interacts with the subcutaneous

$\star$. This work was partially supported by Fondation Sciences Mathématiques de Paris.

$\star \star$. Many thanks to Benoît Perthame for fruitful discussions about this work.

Email address: yannick.deleuze@ljll.math.upmc.fr (Yannick Deleuze). 
loose connective tissue [15]. This manipulation is shown to cause the wrapping of collagen fibers and send a mechanical signal to the extracellular matrix cells (ECM) [14]. There is evidence that needle manipulation in acupuncture causes degranulation of mast calls directly through mechanical stress [17][19]. Mast cells are resident cells in connective tissues, in particular in tissues close to the external environment [18]. They contain many granules rich in chemicals [4]. Mast cells are well known for their role in inflammatory process where they accumulate at the site of inflammation in response to a chemical mediator [16]. However, they also appear to have a protective role [10].

To build our model, we make the assumption that mast cells, when mechanically stressed, release two main kinds of chemicals substances into the ECM : stimulants and chemoattractants. Some stimulants aim at triggering action potential to nearby nerve endings, that can lead for example to liberate opioids and analgesic in the brain [6]. Some stimulants increase the blood vessel lumen as well as its permeability and increase blood flow rate after reaching the heart [12][13]. Then, to maintain a high level of the acupuncture response of mast cells, the chemoattractant participates in cell recruiting of neighbouring mast cells. Recruited mast cells, in turn, degranulate creating a positive feedback process and thus a sustained reaction to acupuncture needling.

Acupuncture as a whole is a complex system. We propose a mathematical model, related to the well known Keller-Segel system for chemotaxis [8], of mast cell response to acupuncture needling close to one acupoint.

\section{Biological model}

We consider the density $n_{g}(t, x)$ of granulated mast cells and the density $n_{d}(t, x)$ of degranulated mast cells around the needle insertion point. Granulated mast cells are stressed by a mechanical stimulus induced by the needle inserted at the acupoint. The function $\Phi(x)$ represents the stimulus signal. The function $\Phi$ is a smooth function of compact support from $\mathbb{R}^{2}$ to $[0,1]$.

When excited, granulated mast cells release into the extracellular environment a chemoattractant, $c(t, x)$, a neural stimulant, $s_{n}(t, x)$, and a endocrine stimulant, $s_{e}(t, x)$. Then, degranulated mast cells can regenerate their chemical mediators to become granulated mast cells again. We consider the release of chemical mediators as quasi-instantaneous and we neglect the transport by convection due to Stokes flow of the matrix fluid created by the motion of the needle.

The following system of partial differential equations in a domain $\Omega$ is a model to describe the mast cell response to acupuncture needling close to one acupoint :

$$
\left\{\begin{array}{l}
\frac{\partial n_{g}}{\partial t}-\mathcal{D}_{m} \nabla^{2} n_{g}+\nabla \cdot\left(\mathrm{S} n_{g} \nabla c\right)=-\mathrm{A} \Phi n_{g}+k_{r} n_{d}, t>0, x \in \Omega \\
\frac{\partial c}{\partial t}-\mathcal{D}_{c} \nabla^{2} c=\kappa_{c} \mathrm{~A} \Phi n_{g}-\delta_{c} c \\
\frac{\partial n_{d}}{\partial t}-\mathcal{D}_{m} \nabla^{2} n_{d}=\mathrm{A} \Phi n_{g}-k_{r} n_{d} \\
\frac{\partial s_{n}}{\partial t}-\mathcal{D}_{s_{n}} \nabla^{2} s_{n}=\kappa_{n} \mathrm{~A} \Phi n_{g}-\delta_{s_{n}} s_{n} \\
\frac{\partial s_{e}}{\partial t}-\mathcal{D}_{s_{e}} \nabla^{2} s_{e}=\kappa_{e} \mathrm{~A} \Phi n_{g}-\delta_{s_{e}} s_{e}
\end{array}\right.
$$

with initial conditions $\left.n_{g}\right|_{t=0}=n_{g}^{0},\left.n_{d}\right|_{t=0}=n_{d}^{0},\left.c\right|_{t=0}=c^{0},\left.s_{n}\right|_{t=0}=s_{n}^{0},\left.s_{e}\right|_{t=0}=s_{e}^{0}$. All the parameters are constants. $S>0$ is the sensitivity of the mast cells to the chemoattractant. $A$ is the activation rate i.e., the rate of mass cells subjected to the mechanical stress $\Phi$ that will degranulate. $\mathcal{D}_{m}, \mathcal{D}_{c}, D_{s_{n}}$ and 
$D_{s_{e}}$ are the diffusion coefficients. $\delta_{c}, \delta_{s_{n}}$ and $\delta_{s_{e}}$ are the degradation rate. $\kappa_{c}, \kappa_{n}$ and $\kappa_{e}$ are the release quantity coefficients of chemoattractant and stimulants. $k_{r}$ is the regeneration coefficient of degranulated mast cells.

This model differs from another chemotaxis model since the chemoattractant is only emitted close to the needle where the cells are mechanically stressed.

\section{Blow-up in the case of only one state for mast cells}

To verify our model (1), we show that blow-up of the density of mast cells occurs when the initial density of mast cells is higher at acupoint than those at nonacupoint locations like what $\mathrm{Yu}$ et al. observed in [17]. Blow-up corresponds to the aggregation of the mast cells close to the treated acupoint. To analyse mathematically the behaviour of our model, we first make some simplifications. From (1), we now consider the evolution of the granulated mast cell density $n$ and the instantaneous diffusion of the chemoattractant

$c$. We avoid the effects of boundary conditions and consider the system (2) in the full space $\mathbb{R}^{2}$. We also consider the stress function $\Phi$ to be equally distributed in a region close to the needle, in other words, for $|x| \leq \lambda, \Phi(x)=1$. Those assumptions lead to the following system:

$$
\left\{\begin{array}{l}
\partial_{t} n-\mathcal{D}_{m} \nabla^{2} n+\nabla \cdot(\mathrm{S} n \nabla c)=-\mathrm{A} \Phi(x) n, t>0, x \in \mathbb{R}^{2}, \\
-\nabla^{2} c=\kappa_{c} \mathrm{~A} \Phi(x) n, \\
\left.n\right|_{t=0}=n^{0} \geq 0 .
\end{array}\right.
$$

Since the initial condition $n^{0}$ is nonnegative, the parabolic equation on $n$ in (2) gives nonnegative solutions. Then, we quantify the spatial distribution of the cells by considering both of the total number of cells and the second moment

$$
m_{0}(t):=\int_{\mathbb{R}^{2}} n(t, x) d x \quad m_{2}(t):=\int_{\mathbb{R}^{2}} \frac{|x|^{2}}{2} n(t, x) d x
$$

Theorem 3.1 In $\mathbb{R}^{2}$, let $n^{0} \in L_{+}^{1}\left(\mathbb{R}^{2},\left(1+|x|^{2}\right) d x\right)$. Let $n$ be a non-negative smooth solution of (2) and let $\left[0, T^{*}\right)$ be the maximal interval of existence. Then, if the initial mass $m_{0}(0)$ is large enough and the second momentum $m_{2}(0)$ is small enough (to be precised in the proof), the solution blows-up as $t \rightarrow T^{*}$.

Proof. The proof follows an argument previously introduced by Nagai in [9], and used in [3] and [11]. First step: total mass. Taking the time derivative on (3) and using (2), we get

$$
\frac{d}{d t} m_{0}(t)=\int_{\mathbb{R}^{2}}-\mathrm{A} \Phi(x) n(t, x) d x \geq-\mathrm{A} m_{0}(t) .
$$

Then,

$$
\forall t \leq T^{*}, m_{0}(0) \geq m_{0}(t) \geq m_{0}(0) \mathrm{e}^{-\mathrm{AT}}
$$

Second step: second moment. Taking the time derivative on (3) and using (2), we get 


$$
\frac{d}{d t} m_{2}(t)=\int_{\mathbb{R}^{2}} \frac{|x|^{2}}{2}\left[\mathcal{D}_{m} \nabla^{2} n(t, x)-\nabla \cdot(\mathrm{S} n(t, x) \nabla c(t, x))-\mathrm{A} \Phi(x) n(t, x)\right] d x .
$$

The second equation in (2) gives us $\nabla c(t, x)$ that we substitute back in the equation. From (5), we get the inequality

$$
\frac{d}{d t} m_{2}(t) \leq 2 \mathcal{D}_{m} m_{0}(t)\left(1-\frac{\mathrm{AS} \kappa_{c}}{8 \pi} m_{0}(t)\right)+\frac{1}{2 \pi} \mathrm{AS} \kappa_{c} \int_{\mathbb{R}^{2}} \int_{\mathbb{R}^{2}} x \frac{x-y}{|x-y|^{2}}(1-\Phi(y)) n(t, y) n(t, x) d y d x .
$$

We can assume that $\Phi$ in a $k_{\Phi}$-Lipchitz function and given $|x| \leq \frac{\lambda}{2}$ and $|y|>\lambda$, then $|x-y| \geq \frac{\lambda}{2}$ i.e. $\frac{1}{|x-y|} \leq \frac{2}{\lambda}$. Moreover, $(1-\Phi(y))$ is bounded from above by 1 and $(1-\Phi(y))=0$ for $|y| \leq \lambda$. This properties of the function $\Phi$ lead to

$$
\frac{d}{d t} m_{2}(t) \leq 2 \mathcal{D}_{m} m_{0}(0)\left(1-\frac{\mathrm{AS} \kappa_{c}}{8 \pi} m_{0}(0) \mathrm{e}^{-\mathrm{AT}} \mathrm{T}^{*}\right)+\frac{3 \sqrt{2}}{2 \pi} \frac{\mathrm{AS} \kappa_{c}}{\lambda}\left(m_{2}(t)\right)^{\frac{1}{2}}\left(m_{0}(0)\right)^{\frac{3}{2}}+\frac{1}{\pi} \frac{\mathrm{AS} \kappa_{c}}{\lambda} k_{\Phi} m_{2}(t) m_{0}(0) .
$$

Finally, following the arguments from [5], we have the following inequation

$$
m_{2}(t) \leq m_{2}(0)+\int_{0}^{t} f\left(m_{2}(s)\right) d s
$$

where $f(\xi)=2 \mathcal{D}_{m} m_{0}(0)\left(1-\frac{\mathrm{AS} \kappa_{c}}{8 \pi} m_{0}(0) \mathrm{e}^{-L T^{*}}\right)+\frac{3 \sqrt{2}}{2 \pi} \frac{\mathrm{AS} \kappa_{c}}{\lambda} \xi^{\frac{1}{2}} m_{0}(0)^{\frac{3}{2}}+\frac{1}{\pi} \frac{\mathrm{AS} \kappa_{c}}{\lambda} k_{\Phi} m_{0}(0) \xi . f$ is a strictly increasing function and if $m_{0}(0)>\frac{8 \pi}{\mathrm{AS} \kappa_{c}} \mathrm{e}^{L T^{*}}$, it exist a unique $\xi^{*}$ such that $f\left(\xi^{*}\right)=0$.

So, if $m_{2}(0)<\xi^{*}$, then $f\left(m_{2}(0)\right)<0$. Therefore, $\int_{0}^{t} f\left(m_{2}(s)\right) d s<0$ and $m_{2}(t) \leq m_{2}(0)+t f\left(m_{2}(0)\right)$ provided that (6) holds true. The second moment becomes nonpositive for $T^{*} \geq-\frac{m_{2}(0)}{f\left(m_{2}(0)\right)}$ which is impossible since $n$ is nonnegative. Therefore, a singularity appears before that time and the solution $n$ blows-up at the singularity point. This can only be appearance of a singular measure because these manipulations hold for correctly defined $L^{1}$ solutions (see [3]).

\section{Existence in the case of only one state for mast cells}

Considering the simplified model (2), we prove the following $L^{p}$ a priori estimate.

Theorem 4.1 In $\mathbb{R}^{2}$, let $p>1$ and assume that $n^{0} \in L_{+}^{1}\left(\mathbb{R}^{2},\left(1+|x|^{2}\right) d x\right)$. There exists a constant $\alpha$ such that when the initial data satisfies $m_{0}(0)<\frac{4 \alpha}{p \mathrm{AS} \kappa_{c}}$, there exists a weak solution to (2) in $L^{p}\left(\mathbb{R}^{2}, d x\right)$ for all times.

Proof. We derive estimates based on the Sobolev inequalities following the argument in [7].

Multiplying (2) by $n^{p-1}$ and integrating, we get

$$
\frac{1}{p} \int_{\mathbb{R}^{2}} \frac{d n^{p}}{d t}=-\int_{\mathbb{R}^{2}} \frac{4(p-1)}{p^{2}}\left|\nabla n^{p / 2}\right|^{2}+\frac{p-1}{p} \int_{\mathbb{R}^{2}} \mathrm{AS} \kappa_{c} \Phi(x) n^{p+1}-\int_{\mathbb{R}^{2}} \mathrm{~A} \Phi(x) n^{p} .
$$

To estimate the integral with power $p+1$, we use the following Gagliardo-Nirenberg-Sobolev inequality 


$$
\int_{\mathbb{R}^{2}}|n|^{p+1} \leq C_{G N S} \int_{\mathbb{R}^{2}}|n| \int_{\mathbb{R}^{2}}\left|\nabla n^{p / 2}\right|^{2} .
$$

Recalling that $\Phi(x) \leq 1$ and $m_{0}(t) \leq m_{0}(0)$,

$$
\frac{1}{p} \int_{\mathbb{R}^{2}} \frac{d n^{p}}{d t} \leq \frac{p-1}{p}\left(-\frac{4}{p}+C_{G N S} \mathrm{AS} \kappa_{c} m_{0}(0)\right) \int_{\mathbb{R}^{2}}\left|\nabla n^{p / 2}\right|^{2}-\int_{\mathbb{R}^{2}} \mathrm{~A} \Phi(x) n^{p} .
$$

Then, if $m_{0}(0)<\frac{4}{p C_{G N S A S} \kappa_{c}}, \int_{\mathbb{R}^{2}} n^{p} d x$ decays in time. From this a priori estimate, we may conclude the existence as done in [11].

\section{Numerical tests}

In this section, we will consider $k_{r}=0$ and thus focus on the two first equation of the system (1). Numerical simulations are carried out using the FreeFem++ software [2]. In the present study, we use one-degree polynomial $\left(\mathrm{P}_{1}\right)$ Lagrangian finite element type in space with a fully implicit Euler scheme in time. Let $V_{h}$ be the corresponding space of piecewise linear continuous functions. The formulation of the problem is the following: Find $n_{g}^{m+1} \in V_{h}$ and $c^{m+1} \in V_{h}$ such that for all $w_{1}, w_{2} \in V_{h}$

$$
\begin{aligned}
& \int_{\Omega} \frac{1}{d t}\left(n_{g}^{m+1}-n_{g}^{m}\right) w_{1}+\int_{\Omega} \mathcal{D}_{m} \nabla n_{g}^{m+1} \nabla w_{1}-\int_{\Omega} \mathrm{S} n_{g}^{m+1} \nabla c^{m} \nabla w_{1}=-\int_{\Omega} \mathrm{A} \Phi(x) n_{g}^{m+1} w_{1}, \\
& \int_{\Omega} \frac{1}{d t}\left(c^{m+1}-c^{m}\right) w_{2}+\int_{\Omega} \mathcal{D}_{c} \nabla c^{m+1} \nabla w_{2}=\int_{\Omega} \kappa_{c} \mathrm{~A} \Phi(x) n_{g}^{m+1} w_{2}-\int_{\Omega} \delta_{c} c w_{2},
\end{aligned}
$$

At each step $m$, we have a non linear problem to solve. We use the fixed-point technique. To gain time, mesh adaptation (a subroutine of FreeFem ++ ) fits the initial condition, i.e., a given cell distribution within the domain of interest, as the solution evolves locally. We apply Neumann boundary conditions (no cell and chemoattractant fluxes) at the border of the computational domain.

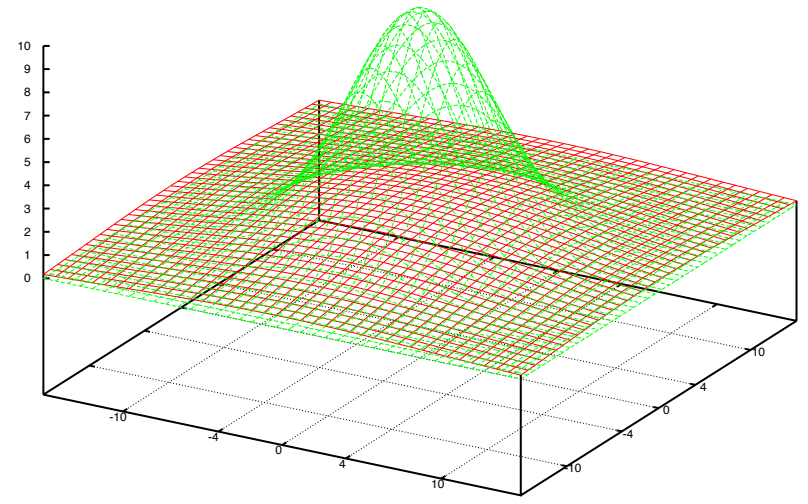

Figure 1. Initial mast cell Gaussian distribution in an acupoint (concentrated distribution) and non-acupoint (dispersed distribution) with the same cell number.

According to the initial distribution of the mast cell distribution [Fig. 1 ] on the one hand and to the needle position with respect to the location of the peak cell density on the other, i.e., whether the 

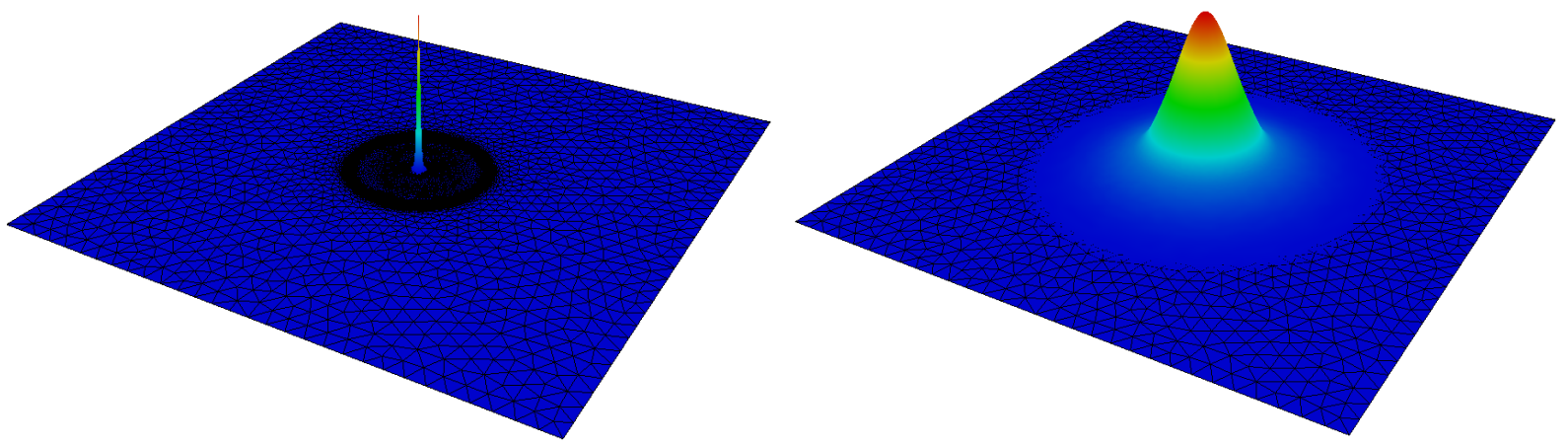

Figure 2. Final distribution of mast cells at acupoint (left) and in a non-acupoint mast cell pool (right). Expected blow-up solution is only achieved at acupoint.
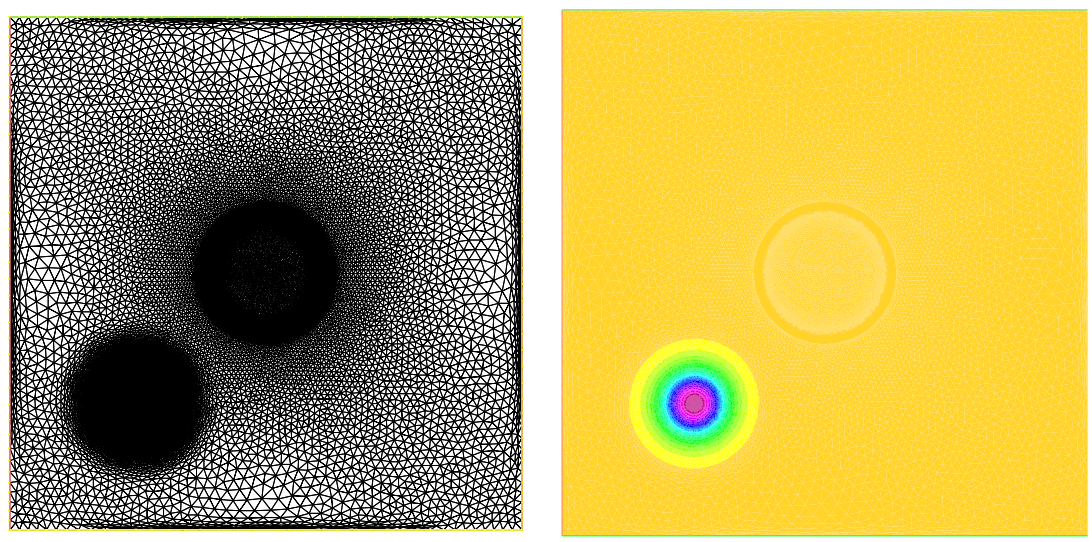

Figure 3. Needling outside an acupoint. Mesh with refinements in the needle region (center) and mast cell pool (left bottom corner). Absence of significant change in cell population distribution.

practitioner is an expert or not, the expected blow up' solution is obtained [Fig. 2 (left)] or not [Fig. 2 (right), Fig. 3]

\section{Conclusion}

With the mathematical analysis of a simplified model we have shown a mechanism for blow-up in the chemotactic mechanism involved during acupuncture. A small second moment of the density of mast cells convey the idea that the cells are concentrated at the acupuncture points. Blow-up will occur when the initial number of mast cells is high enough and that they are sufficiently concentrated around the acupuncture points. Our model behaviour corresponds to the behaviour expected for the response of mast cells to acupuncture needling : a response of the mast cells exists when their density is low, for instance on a non-acupoint, but is greater when the initial density of mast cells is higher on a acupoint. In that case, the aggregation of mast cells could reveals the efficiency of the needle manipulation. We provide a numerical validation of the mathematical model of mast cell response to acupuncture needling. 


\section{References}

[1] X. Cheng, Chinese acupuncture and moxibustion, 1st ed., Foreign Language Press, Beijing, 1987.

[2] F. Hecht, New Development in FreeFem++; J. Numer. Math., Vol. ?, No. ?, pp. 620 (2013), to appear.

[3] B. Perthame, Transport Equations in Biology, Birkhuser, 2007.

[4] M. Thiriet, Biology and Mechanics of Blood Flows: Part I: Biology, Springer New York, 2008.

[5] V. Calvez, L. Corrias, The Parabolic-Parabolic Keller-Segel Model in R2, Commun. Math. Sci. 6 (2008) $417-447$.

[6] S.-H. Hsiao, L.-J. Tsai, A Neurovascular Transmission Model for Acupuncture-induced Nitric Oxide, Journal of Acupuncture and Meridian Studies. 1 (2008) 42-50.

[7] W. Jager, S. Luckhaus, On Explosions of Solutions to a System of Partial Differential Equations Modelling Chemotaxis, Transactions of the American Mathematical Society. 329 (1992) pp. 819-824.

[8] E.F. Keller, L.A. Segel, Model for chemotaxis, Journal of Theoretical Biology. 30 (1971) 225-234.

[9] T. Nagai, T. Senba, Global Existence and Blow-up of Radial Solutions to a Parabolic-Elliptic System of Chemotaxis, Advances in Mathematical Sciences and Applications. 8 (1998) 145156.

[10] M. Urb, D.C. Sheppard, The Role of Mast Cells in the Defence against Pathogens, PLoS Pathog. 8 (2012) e1002619.

[11] A. Blanchet, J. Dolbeault, B. Perthame, Two-dimensional Keller-Segel model: Optimal critical mass and qualitative properties of the solutions, Electronic Journal of Differential Equations. 44 (2006) 1-33.

[12] H. Hsiu, W.-C. Hsu, C.-L. Hsu, S.-M. Huang, Assessing the effects of acupuncture by comparing needling the hegu acupoint and needling nearby nonacupoints by spectral analysis of microcirculatory laser Doppler signals, Evid Based Complement Alternat Med. 2011 (2011) 435928.

[13] T.C. Kuo, Y.J. Chen, H.Y. Kuo, C.F. Chan, Blood Flow Effect of Acupuncture on the Human Meridian, Medical Acupuncture. 22 (2010) 33-40.

[14] H.M. Langevin, D.L. Churchill, J. Wu, G.J. Badger, J.A. Yandow, J.R. Fox, et al., Evidence of connective tissue involvement in acupuncture, FASEB J. 16 (2002) 872-874.

[15] H.M. Langevin, D.L. Churchill, M.J. Cipolla, Mechanical signaling through connective tissue: a mechanism for the therapeutic effect of acupuncture, FASEB J. 15 (2001) 2275-2282.

[16] G. Nilsson, M. Johnell, C.H. Hammer, H.L. Tiffany, K. Nilsson, D.D. Metcalfe, et al., C3a and C5a Are Chemotaxins for Human Mast Cells and Act Through Distinct Receptors Via a Pertussis Toxin-Sensitive Signal Transduction Pathway, The Journal of Immunology. 157 (1996) 1693-1698.

[17] X. Yu, G. Ding, H. Huang, J. Lin, W. Yao, R. Zhan, Role of Collagen Fibers in Acupuncture Analgesia Therapy on Rats, Connective Tissue Research. 50 (2009) 110-120.

[18] D. Metcalfe, D. Baram, Y. Mekori, Mast cells, Physiological Reviews. 77 (1997) 1033-1079.

[19] D. Zhang, G. Ding, X. Shen, W. Yao, Z. Zhang, Y. Zhang, et al., Role of Mast Cells in Acupuncture Effect: A Pilot Study, Explore (NY). 4 (2008) 170-177. 